\title{
Author Index 2012
}

A

Adhikari S. (See Pal A.)

Aditya V. (See Meenakshi)

Agarwal A. See Pandey K.

Agarwal N. See Pandey K.

Aggarwal A. (See Kaur T.)

Aggarwal R., Suneja A., Vaid N.B., Yadav P., Sharma A.,

Mishra K. . . . . . . . . . . . . . . . . . 77

Al Tajir G. (See Khan M.J.)

Ali G. (See Khan M.J.)

Allahbadia G.N. . . . . . . . . . . . . . . . . . . . . 381

Anklesaria B. . . . . . . . . . . . . . . . . . . . 209

Arora R.(See Nirmala C.)

Arora U. (See Jindal N.)

Aruna P. (See Kumar M.)

Avasthi K. (See Juneja S.K.)

Avraham S., Seidman D.S.

\section{B}

Badry A.A. (See Shalaby H.A.R.)

Bal. S.M. (See Goyal R.)

Balasubramaniam A. (See Dewan B.)

Balkawade N.U., Shinde M.S. . . . . . . . . . . . 520

Balsarkar G. (See Purandare C.N.)

Balwan W.K. (See Sharma S.)

Banerjee S. (See Dam P.)

Bansal S. (See Kaur T.)

Batra S., Nayak H., Dave K.S. . . . . . . . . . . . 541

Begum N. (See Mukhopadhyay P.)
Behbahani F. (See Saleh G.)

Behtash N. (See Saleh G.)

Belli A.M. (See Talaulikar V.S.)

Bhagoliwal A. (See Pandey K.)

Bhanja C.K. (See Roy C.)

Bhanja Chowdhury M. (See Dasgupta M.)

Bhansali R.P. (See Nirmala C.)

Bhardwaj N., (See Sharma B.)

Bhat M., Ramesha K.N., Sarma S.P., Menon S.,

Ganesh K.S. . . . . . . . . . . . . . . . . . . . 644

Bhattacharya I.K. (See Mahanta R.)

Bhattacharya P. (See Mukhopadhyay P.)

Bhattacharya S.K. (See Kamliya G.)

Bhattarcharya S.K., Kundu S., Kabiraj S.P. . . . . . . 297

Bhosale A., Fonseca M. . . . . . . . . . . . . . . . . 197

Bhute S. (See Shrivastava D.)

Bindu H. (See Lingeswaran A.)

Biswas B.N. (See Dasgupta M.)

Biswas S.C. (See Dey R.)

C

Chakravarty C., Sivakumaran S. Punk J., Singh N.,

Pandey R., Darlong V. . . . . . . . . . . . . 205

Chakravorty P.S. (See Tibrewal R.)

Chanam M.S. (See Khumanthem P.D.)

Chandalia H.B. (See Hinduja I.N.)

Chandel L.R., Kanga A., Chauhan S. . . . . . . . . . 511

Chatterjee S. (See Dasgupta M.)

Chattopadhyay N. (See Dey R.) 
Chaturvedi V. (See Jain D.)

Chaudhary A. (See Chaudhary A.K.)

Chaudhary A., (See Konar H.)

Chaudhary A.K., Chaudhary A., Tiwary S.C.,

Dwivedi R. . . . . . . . . . . . . . 286

Chaudhury P.N.B. (See Mahanta R.)

Chauhan A. (See Nirmala C.)

Chauhan M., Kala J. . . . . . . . . . . . . . . . . . . . . . 442

Chauhan S. (See Chandel L.R.)

Chowdhury G., Sinth N. . . . . . . . . . . . . . . . 192

Chowdhury K. (See Roy C.)

Chibber P.J. (See Hinduja I.N.)

D

Dadhwal V., (See Deka D.)

Dadhwal V., (See Deka D.)

Dam P, Saha S.K. Banerjee S., Ganduly S. . . . . . 334

Darlong V. (See Chakravarty C.)

Das B., Saha Roy S., Das Gupta A., Lahiri T.K.,

Das N.N. . . . . . . . . . . . . . . . . . . . 59

Das H.N. (See Das B.)

Dasgupta A. (See Das B.)

Dasgupta E., Singh G. . . . . . . . . . . . . . 67

Dasgupta M. (See Pal A.)

Dasgupta M., Biswas B.N., Chatterjee S., Mazumdar P.,

Bhanja Chowdhury M. . . . . . . . . . . . . . . 419

Dave A., Mathur P., Mathuriya G. . . . . . . . . . . 687

Dave K.S. (See Batra S.)

Dave K.S. (See Nirmala C.)

Dave K.S. (See Patel S.M.)

Deka D., Dadhwal V., Gajatheepan S.B., Singh A.,

Sharma K.A., Malhotra N. . . . . . . . . . . . . 655

Deka D., Dadhwal V., Roy Kumar K., Malhotra N.,

Vaid A., Mittal S. . . . . . . . . . . . . . 40

Desai A.D. (See Patel S.M.)

Devaru D., Thusoo M. . . . . . . . . . . . 63

Devi B. (See Jindal N.)

Dewan B., Philipose N., Balasubramanian A. . . . . 281

Dewangan R. (See Singh A.)

Dey R., Biswas S.C., Chattopadhyay N., Gupta D.,

Roy Biswas R., Mukhopadhyay A. . . . . . . . . . 332

Dhand H.C. (See Dave A.)

Dhariwal V. (See Kumar M.)

Dhawan S. (See Kaur T.)

Dhuhan N., Kadian Y.S., Sirohiwal D., Pradeep K.,

Nidhi R. . . . . . . . . . . . . . . . . . . . . . . 446

Divya A. (See Jassal C.D.)

Diwedi R. (See Chaudhary A.K.)

Doshi H. (See Shah K.)

Dubay P. (See Pandey K.)
$\mathbf{E}$

Elhady R.A. (See Shalaby H.A.R.)

F

Fomulu J.N. (See Nkwabong E.)

Fonseca M. (See Bhosale A.)

G

Gajatheepan S.B. (See Deka D.)

Gamal A.M. (See Shalaby H.A.R.)

Ganesh K.S. (See Bhat M.)

Ganguly S. (See Dam P.)

Garg S. (See Juneja S.K.)

Ghosh A. (See Roy C.)

Gill K., Majumdar A. 195

Girija S. (See Manjunath A.P.)

Gogoi A. (See Mahanta R.)

Goswami S. (See Tibrewal R.)

Goyal R., Mohi K.M., Bal S.M. . . . . . . . . . . . . 437

Guin G., Sahu B., Khare S., Kavishvar A. . . . . . . . 307

Guin G., Shaw K., Khare S. . . . . . . . . . . . 45

Gupta D., (See Dey R.)

Gupta N. (See Pandey K.)

Gupta P.K. (See Sharma B.)

Gupta S. (See Sharma B.)

Gupta S. (See Sharma S.)

\section{$\mathbf{H}$}

Hakim S. (See Mohsin Z.)

Halder A., Mukherjee G., Pati S., Halder S., Nayak R. 327

Halder A., Pati S., Mukherjee G., Halder S. . . . . . . 96

Halder S. (See Halder A.)

Halder S. (See Halder A.)

Handa S.R. (See Hinduja I.N.)

Hansda J., Roychowdhury J. . . . . . . . . . . . . . . . 536

Hazra A. (See Kamliya G.)

Hegde C.V. . . . . . . . . . . . . . . . 21

Hegde C.V. . . . . . . . . . . . . . . . . . 501

Himani S. (See Kaur G.)

Hinduja I.N. Laliwala D.H., Chandalia H.B., Chibber P.J.,

Handa S.R., Khubchandani S.R. . . . . . . . . . . 302

\section{I}

Inamdar S. (See Shrivastava D.)

\section{$\mathbf{J}$}

Jain D., Khuteta R., Chaturvedi V., Khuteta S. . . . 429

Jain V. (See Kaur G.)

Janaki S. (See Pillay P.)

Jarag M.A. (See Modi A.)

Jassal C.D., Patnaik B.L., Divya A., Prasad S. . . . . 93 
Jindal N., Arora U., Singh S., Devi B. . . . . . . . . 158

Juneja S.K., Avasthi K., Malhotra V., Garg S. . . . 689 Jyoti M.(See Kumar M.)

\section{$\mathbf{K}$}

Khare S. (See Guin G.)

Kaur G., Jain V., Mehta S., Himani S. . . . . . . . . . 52

Kamilya G., Seal S.L., Mukherji J., Roy H.,

Bhattacharya S.K., Hazra A. . . . . . . . . . . . 55

Kumar M., Jyoti M., Aruna P., Poddar A., Dhariwal V.,

Shailendra K. . . . . . . . . . . . . . . . 72

Khumanthem P.D., Chanam M.S., Samjetshabam R.D. 168

Kantharia S.L. (See Modi A.)

Kumar P. (See Sharma S.)

Khubchandani S.R. (See Hinduja I.N.)

Khadilkar S. . . . . . . . . . . . . . . . . 261

Kar S., Krishnan A., Shivkumar P.V. . . . . . . . . 268

Krishnan A. (See Kar S.)

Kushwaha K.P. (See Meenakshi)

Kundu S. (See Bhattacharya S.K.)

Kabiraj S.P. (See Bhattacharya S.K.)

Konar H., Chaudhuri S. . . . . . . . . . . . . . . . . 301

Khare S. (See Guin G.)

Kavishwar A. (See Guin G.)

Khuteta S. (See Jain D.)

Kala J. (See Chauhan M.)

Kadian Y.S. (See Duhan N.)

Kaur R., Dhawan S., Aggarwal A., Bansal S. . . . . 450

Kanga A. (See Chandel L.R.)

Kauam L. (See Nkwabonge E.)

Khan M.J., Ali G., Al T.G., Sulieman H. . . . . . . . 660

Kochar S., Prakash P. . . . . . . . . . . . . . . . . . . . 694

L

Lahange K.K. (See Patel S.M.)

Lahiri T.K. (See Das B.)

Lakshmidevi M., Malini K.V, Shetty V.H. . . . . . 635

Laliwala D.H. (See Hinduja I.N.)

Lingeswaran A., Bindu H.

M

Mahanta R., Gogoi A., Chaudhury P.N.B., Roy S.,

Bhattacharya I.K., Sharma P. . . . . . . . . . . . 546

Mahmood T. (See Vladimir R.)

Majumdhar A. (See Gill K.)

Malhotra N. (See Deka D.)

Malhotra V. (See Juneja S.K.)

Malini K.V., (See Lakshmidevi M.)

Malviya K. (See Sharma B.)

Mandal A.K. (See Pal A.)

Manjila C. (See Pillay P.)

Manjunath A.P. Girija S. . . . . . . . . . . . 25

Manyonda I. (See Talaulikar V.S.)

Matah M., Sareen S.
Mathur P. (See Dave A.)

Mathuriya G. (See Dave A.)

Mazumdar P. (See Dasgupta M.)

Meenakshi, Srivastava R., Sharma N.R., Kushwaha K.P.,

Aditya V. . . . . . . . . . . . . . . . . . 276

Mehta S. (See Kaur G.)

Menon S. (See Bhat M.)

Mishra K. (See Aggarwal R.)

Mishra N. (See Singh A.)

Mittal S. (See Deka D.)

Modi A., Moitra M., Verma R., Patel B., Jarag M.A.,

Kantharia S.L. . . . . . . . . . . . . . . . . . . . 184

Mohi K.M. (See Goyal R.)

Mohsin Z. (See Nigar A.)

Moitra M. (See Modi A.)

Mokta Kiran K. (See Chandel L.R.)

Mujalde A. (See Dave A.)

Mukherje J. (See Kamilya G.)

Mukherjee G. (See Halder A.)

Mukhopadhyay A. (See Dey R.)

Mukhopadhyay P., Bhattacharya P., Begum N. . . . 88

Mukhopadhyay S. (See Vladimir R.)

Muralidhar A. (See Vladimir R.)

$\mathbf{N}$

Nagarsekar U. (See Talaulikar V.K.)

Nageshu S. (See Swamy M.K.)

Naik S.A., Patel A.M. Shah J.M., Verma R.N. . . . 448

Nandi L. (Singh A.)

Nayak H. (See Batra S.)

Nayek R. (See Halder A.)

Nidhi R. (See Duhan N.)

Nigar A., Hakim S., Mohsin Z. 398

Nirmala C., Dave K.S., Chauhan A., Bhansali R.P.,

Arora R. . . . . . . . . . . . . . . 322

Nkwabong E., Fomula J.N., Kouam L., Ngassa P.C. 531

Nkwabong E., Kouam L. 641

\section{$\mathbf{P}$}

Pal A., Roy D., Adhikari S., Roy A., Dasgupta M.,

Mandal A.K. . . . . . . . . . . . . . . . . 312

Pal M. (See Roy C.)

Pandey K., Dubay P., Bhagoliwal A., Gupta N.,

Tyagi G. . . . . . . . . . . . . . . 406

Pandey K.,Pradhan A., Agarwal A., Bhagoliwal A.,

Agarwal N. . . . . . . . . . . . . . . 432

Pandey R. (See Chakravarty C.)

Patel A.N. (See Naik S.A.)

Patel B. (See Modi A.)

Patel M.A. (See Purandare C.N.)

Patel M.A. (See Purandare C.N.)

Patel M.A. (See Purandare C.N.)

Patel S.M., Lahamge K.K., Desai A.D., Dave K.S. 176 
Pati S. (See Halder A.)

Pati S. (See Halder A.)

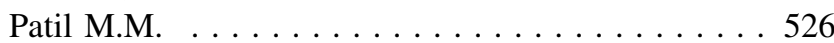

Patnaik B.L. (See Jassal C.L.) . . . . . . . . . . 82

Philipose N. (See Dewan B.) . . . . . . . . . . 266

Pillay P., Janaki S., Manjila C. . . . . . . . . . . . . . . . 409

Poddar A. (See Kumar M.)

Pradeep K. (See Duhan N.)

Pradhan A. (See Pandey K.)

Prakash P. (See Kochar S.)

Prasad S. (See Jassal C.L.)

Premalatha R., Tripathy M.S. . . . . . . . . . . 82

Punk J. (See Chakravarty C.)

Purandare C.N. . . . . . . . . . . . . . . . . . . . . 141

Purandare C.N. . . . . . . . . . . . . . . . . . . 621

Purandare C.N., Patel M.A, Balsarkar G.D. . . . . . . 23

Purandare C.N., Patel M.A, Balsarkar G.D. . . . . . . 141

Purandare C.N., Patel M.A, Balsarkar G.D. . . . . . 266

Purandare C.N., Patel M.A, Balsarkar G.D. . . . . . . 384

Purandare C.N., Patel M.A, Balsarkar G.D. . . . . . . 504

Purandare C.N., Patel M.A, Balsarkar G.D. . . . . . 624

Purandare S.V. (See Shafi D.)

\section{Q}

\section{R}

Ramalingappa A. Yashoda

Ramesha K.N. (See Bhat M.)

Rane A., Iyer J. . . . . . . . . . . . . . . . . . . . . 626

Rangasamy S., Rao A. . . . . . . . . . . . . . . . . 424

Rao A. (See Rangasamy S.)

Raut-Dessai R. (See Tilve A.J.)

Ribeiro R. (See Tilve A.J.)

Roy A. (See Pal A.) . . . . . . . . . . . . . . . . . . . . 179

Roy Chhaya, Bhanja C.K., Pal M., Chowdhury K.,

Ghosh A. . . . . . . . . . . . . . . . . . 679

Roy D. (See Pal A.)

Roy H. (See Kamilya G.)

Roy Kumar K. (See Deka D.)

Roy S. (See Mahanta R.)

Roy S.S., Sengupta S.

Roybiswas R. (See Dey R.)

Roychowdhury J. (See Hansda J.)

S

Saha Roy S. (See Das B.)

Saha S.K. (See Dam P.)

Sahu B. (See Guin G.)

Saleh G., Saraya S., Behrakh B.F., Behtash N.,

Samiei F. . . . . . . . . . . . . . . . . . 674

Samiei F. (See Saleh G.)

Samjetshabam R.D. (See Khumanthem P.D.)
Saraya S. (See Saleh G.)

Sareen S. (See Matah M.)

Sarma S.P. (See Bhat M.)

Sathe A.V. (See Shafi D.)

Sawant B.V. (See Tilve A.J.)

Seal S.L. (See Kamilya G.)

Seidman D.S. (See Avraham S.)

Sengupta S. (See Roy S.S.)

Shafi D., Purandare S.V., Sathe A.V. . . . . . . . . 317

Shah J.M. (See Naik S.A.)

Shah S. . . . . . . . . . . . . . . . . 551

Shailendra K. (See Kumar M.)

Shak K., Doshi H. . . . . . . . . . . . . . . . . . . . 172

Shalaby H.A.R., Elhady R.A., Gamal A.M.,

Badry A.A. . . . . . . . . . . . . . 515

Sharma A. (See Aggarwal R.)

Sharma B., Bhardwaj N., Gupta S., Gupta P.K., Verma A.,

Malviya K. . . . . . . . . . . . . 650

Sharma K.A. (See Deka D.)

Sharma N.R. (See Meenakshi)

Sharma P. (See Mahanta R.)

Sharma S., Balwan W.K., Kumar P., Gupta S. . . . . 199

Shaw K. (See Guin G.)

Shetty V.H. (See Lakshmidevi M.)

Shivkumar P.V. (See Kar S.)

Shrivastava D., Inamdar S., Bhute S., Singh A. . . . . 154

Singh A. (See Deka D.)

Singh A. (See Shrivastava D.)

Singh A., Mishra N., Dewangam P. . . . . . . . . . . 401

Singh A., Nandi L. . . . . . . . . . . . . . . . . . . 291

Singh G. (See Dasgupta E.)

Singh N. (See Chakravarty C.)

Singh N. (See Chowdhury G.)

Singh S. (See Jindal N.)

Singh S. (See Sood A.K.) . . . . . . . . . . . . . 162

Sirohiwal D. (See Huhan N.)

Sivakumaran S.(See Chakravarty C.)

Sood A.K. (See Chandel L.R.)

Sood A.K. (See Singh S.)

Srivastava R. (See Meenakshi)

Suliaman H. (See Khan M.J.)

Suneja A. (See Aggarwal R.)

Swamy M.K., Patil K., Nagashu S. . . . . . . . . . . 413

$\mathbf{T}$

Talaulikar V., Nagarsekar U. . . . . . . . . . . . . . . . 146

Talaulikar V.S., Belli A.N., Manyanda I. . . . . . . . 506

Thakur K. (See Chandel L.R.)

Thusoo M. (See Devaru D.)

Tibrewal R., Goswami S., Chakravorty P.S. . . . . . . 452

Tilve A.J., Ribeiro R., Sawant B.V., Raut Dessai R. 329

Tiwari S.C. (See Chaudhary A.D.)

Tripathi M.S. (See Premalatha R.) 
Tyagi G. (See Pandey K.)

\section{$\mathbf{U}$}

V

Vaid A. (See Deka D.)

Vaid N.B. (See Aggarwal R.)

Verma A. (See Sharma B.)

Verma R. (See Modi A.)
Verma R.N. (See Naik S.A.)

Vladimir R., Muralidhar A., Mukhopadhyay s.,

Mahmood T. .................6665

WXYZ

Yadav P. (See Aggarwal R.)

Yashoda (See Ramalingappa A.) 